\title{
Anisotropy of Hectometric Cosmic Background
}

\author{
Y.V. Tokarev ${ }^{1}$, M.L. Kaiser ${ }^{2}$, G.N. Boiko ${ }^{1}$ and P.V. Gustov ${ }^{1}$ \\ ${ }^{1}$ Radiophysical Research Institute, 25/14 Bolśhaya Pecherskaya st., \\ Nizhny Novgorod, 603600, RF. \\ ${ }^{2} N A S A$, Goddard Space Flight Center, Greenbelt, MD 20771, USA.
}

\begin{abstract}
The results of investigation of the low-frequency cosmic background by the WIND spacecraft near the $L 1$ point during the deepest phase of the last solar activity minimum are presented. The antenna temperature modulation index and the ecliptic longitude of the primary area of radiation for frequencies $260,516,772$, and $1028 \mathrm{kHz}$ were measured by means of a dipole on the spacecraft which is rotated in ecliptic plane. Our results correct and complement the conclusions of the previous measurements made with IMP-6.
\end{abstract}

\section{Introduction}

Till recently, the ultra-low-frequency data on cosmic radio emission at frequencies below $0.5 \mathrm{MHz}$ was limited to those obtained more than 25 years ago using the IMP-6 and RAE-2 spacecrafts. Sporadic solar and terrestrial interference is a serious source of error and contamination in this data. The problem of terrestrial interference (Terrestrial Kilometric Radiation) is much less for the WIND spacecraft whose orbit apogee is as large as 1.5 million kilometres. The WAVES RAD1 receiver of the WIND spacecraft was designed to study radio and plasma wave phenomena in the range $20-1040 \mathrm{kHz}$ (Bougeret and Kaiser, 1995). It is equipped with $100-\mathrm{m}$ dipole antenna rotating in the ecliptic plane with a period of $3.00169 \mathrm{~s}$ and it is possible to measure the angle between sun's direction and antenna axis.

The observation were made during 16-30 December 1997 near the winter solstice when the WIND was was about $1.4 \mathrm{~m} \mathrm{~km}$ from the Earth. The background intensity was measured in series at $260,512,772$ and $1028 \mathrm{kHz}$ with sampling period of $1.432 \mathrm{sec}$. The measurements at $260 \mathrm{kHz}$, which were more noisy, were repeated on 24-28 January, 02-05 April and 04-10 October 1998 with a sampling frequency of 0.3583 sec.

\section{Measurement Results}

The measured modulation index $m$ and ecliptic longitude $L_{\max }$ of the effective galactic source at frequencies are given in Table 1 as well as in Fig. 1.

The modulation index $m$, at frequencies between $500-1000 \mathrm{kHz}$ are within the measured errors, comparable to those found by Brown (1973). But instead 


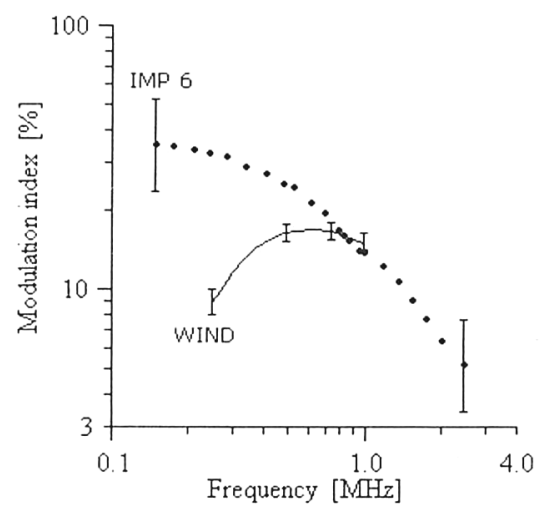

\begin{tabular}{ccc}
\multicolumn{3}{c}{ Table I } \\
\hline$f(\mathrm{kHz})$ & $m(\%)$ & $L_{\max }(\mathrm{deg})$ \\
\hline 260 & $9 \pm 1$ & $185 \pm 3$ \\
516 & $17 \pm 1$ & $179 \pm 2$ \\
772 & $17.3 \pm 1$ & $177 \pm 1$ \\
1028 & $16.3 \pm 1$ & $173 \pm 1$ \\
\hline
\end{tabular}

Figure 1. Comparision of IMP-6 and WIND measurements of modulation index. Table gives measured values.

of a monotonic increase of $m$ with the decrease of $f$ we find a weak maximum near $f=600 \mathrm{kHz}$ (Fig. 1).

Our determination of the direction of the radiation is close to that determined by Brown (1973) for $f=600 \mathrm{kHz}$. He noted that the area of the increased radio emission was localized at a angular distance of $30^{\circ}$ from the galactic pole. The $180^{\circ}$-uncertainty in dipole antenna observations does not allow us to eliminate either of two possible opposite directions. However the RAD1 observations show an interesting trend of Lmax with the observation frequency.

Favorable observing conditions of WIND spacecraft - low spurious Earth and solar sporadic radiation occurences, a large distance from Earth and the possibility of observing the galactic "radio source" at different elongations - have provided more reliable new data on angular anisotropy of hectometric cosmic background. The new data on the modulation index and predominant arrival angles provide strong constraint on models that try to explain the cosmic background radiation (Getmantsev,1965, Bougeret et al, 1984, Steinberg et al, 1986).

Acknowledgments. This work was supported by RFBR (grant No 99-0217285) and INTAS (grant No 97-1964).

\section{References}

Brown, L.W., 1973, Astrophys.J.,180, 359

Bougeret J.-L., Kaiser, M.L. Kellogg P.J., et al, 1995, Space Sci. Rev.,71,231.

Bougeret J-L., Fainberg J., and Stone R.G., 1984, Astron. Astroph.,136,17.

Getmantsev G.G., 1965, Kosm. Issled.,3, 495. (in Russian)

Steinberg J-L., Fainberg J., Meyer-Vernet N. and Hoang S., 1986, Geophys. Res. Letters, $13,407$. 